Systematic review

\title{
The association between antimicrobial resistance and HIV infection: a systematic review and meta-analysis
}

\author{
Ioana D. Olaru 1,2, *, Evelina Tacconelli ${ }^{3}$, Shunmay Yeung ${ }^{1}$, Rashida A. Ferrand ${ }^{1,2}$, \\ Richard A. Stabler ${ }^{1}$, Heidi Hopkins ${ }^{1}$, Alexander M. Aiken ', Katharina Kranzer 1, 2,'4 \\ 1) Clinical Research Department, Faculty of Infectious and Tropical Diseases, London School of Hygiene and Tropical Medicine, London, United Kingdom \\ 2) Biomedical Research and Training Institute, Harare, Zimbabwe \\ 3) Infectious Diseases, Department of Diagnostic and Public Health, University of Verona, Verona, Italy \\ ${ }^{4)}$ Division of Infectious and Tropical Medicine, Medical Centre of the University of Munich, Munich, Germany
}

\section{A R T I C L E I N F O}

\section{Article history:}

Received 5 October 2020

Received in revised form

23 February 2021

Accepted 14 March 2021

Available online $\mathrm{xxx}$

Editor: M. Leeflang

\section{Keywords:}

AIDS

AMR

Antibiotic resistance

Bacterial infections

Colonization

HIV

\begin{abstract}
A B S T R A C T
Objectives: People living with HIV (PLWH) are at increased risk of infections with resistant organisms due to more frequent healthcare utilization. Our objective was to investigate the association between HIV and antimicrobial resistance (AMR).

Methods: We searched MEDLINE, EMBASE, Web of Science, LILACS and African Journals Online. Studies were eligible if they reported on AMR for colonization or infection with bacterial pathogens (excluding mycobacteria and bacteria causing sexually transmitted infections) and were stratified by HIV status, species and antimicrobials tested. Pooled odds ratios were used to evaluate the association between HIV and resistance.

Results: In total, 92 studies published between 1995 and 2020 were identified. The studies included the following organisms: Staphylococcus aureus $(n=47)$, Streptococcus pneumoniae $(n=28)$, Escherichia coli $(n=6)$ and other Gram-negative bacteria. PLWH had a 2.12 (95\%CI 1.36-3.30) higher odds for colonization and 1.90 (95\%CI 1.45-2.48) higher odds for infection with methicillin-resistant S. aureus, a 2.28 (95\%CI 1.75-2.97) higher odds of infection with $S$. pneumoniae with decreased penicillin susceptibility, and a 1.59 (95\% CI $0.83-3.05)$ higher odds of resistance to third-generation cephalosporins in E. coli and Klebsiella pneumoniae.

Conclusion: This review shows an increased risk of AMR in PLWH across a range of bacterial pathogens and multiple drug classes. The lack of laboratory capacity for identifying AMR, and limited access to alternative treatment options in countries with the highest burden of HIV, highlight the need for more research on AMR in PLWH. Overall, the quality of studies was moderate or low, which may impact the findings of this review. Ioana D. Olaru, Clin Microbiol Infect 2021;n:1

(C) 2021 The Authors. Published by Elsevier Ltd on behalf of European Society of Clinical Microbiology and Infectious Diseases. This is an open access article under the CC BY license (http://creativecommons.org/
\end{abstract} licenses/by/4.0/).

\section{Introduction}

The discovery of antimicrobials has led to a 'golden age' in medicine which has enabled the effective treatment of a large number of once deadly infections. The emergence and worldwide dissemination of antimicrobial resistance (AMR) is threatening to overturn these advances, leading to increased morbidity, mortality and healthcare costs [1]. AMR is of particular concern in low- and

\footnotetext{
* Corresponding author: Ioana D. Olaru, London School of Hygiene and Tropical Medicine, Keppel Street, Bloomsbury, London, WC1E 7HT, United Kingdom.

E-mail address: ioana-diana.olaru@lshtm.ac.uk (I.D. Olaru).
}

middle-income settings where treatment options are limited due to cost and availability of drugs. Among the main drivers for AMR are inappropriate antimicrobial use, transmission of resistant pathogens in healthcare settings, and rapid dissemination of resistant pathogenic strains due to international travel and trade [2]. On an individual level, prior antimicrobial use, healthcare contact and underlying comorbid conditions are recognized risk factors for infection and colonization with resistant organisms [3].

There are almost 38 million people living with HIV (PLWH) worldwide, with the highest prevalence of infection in countries in sub-Saharan Africa [4]. PLWH have more frequent hospital admissions, clinic visits and antimicrobial treatment courses compared to 
individuals without HIV infection, putting them at increased risk of the acquisition of and infection with resistant bacteria. Hospital admissions for acute bacterial infections are more frequent in HIVinfected than in HIV-uninfected individuals [5,6]. Overall, bacterial infections (not including tuberculosis) are one of the main causes of hospitalization and death in PLWH worldwide, with almost a third of all hospital admissions and a quarter of all deaths due to bacterial infections [7]. More frequent hospital admissions may lead to an increased number of antimicrobial prescriptions and a higher risk of acquisition of resistant pathogens during hospitalization. Additionally, there is a higher prevalence of chronic conditions such as kidney and liver diseases, which also require more frequent clinic appointments and hospital admissions among PLWH [8,9]. In the United States, prescriptions for multiple antimicrobial drug classes are more common among elderly HIV-infected individuals than in their HIV non-infected counterparts [10]. Furthermore, antimicrobial prescriptions are more frequent among HIV-infected individuals even before HIV diagnosis, as shown by a study from Denmark [11]. With the roll-out of widespread antiretroviral therapy (ART) and improved early diagnosis and treatment of HIV, the impact of these factors on the prevalence of resistance may have changed over time.

Antimicrobials such as co-trimoxazole, macrolides and antimycobacterial agents are also recommended as prophylaxis for PLWH $[12,13]$. The indication may vary by CD4 cell count, age, geographical setting and prior medical history. Use of cotrimoxazole prophylaxis in high-income settings is decreasing due to early diagnosis of HIV and ART use. However, in low-income settings co-trimoxazole prophylaxis is recommended by the WHO for all children with HIV, and for adults with advanced HIV infection or who are living in areas where malaria and bacterial infections are prevalent, irrespective of ART [12]. Thus, co-trimoxazole prophylaxis, which has significantly reduced mortality [14], is currently widely used throughout sub-Saharan Africa.

Although HIV infection is not known to have a direct effect on the colonization and infection of an individual with resistant organisms, PLWH have a multitude of indirect risk factors. There are limited data on the association between HIV infection and bacterial AMR, and to our knowledge this has never been reviewed systematically.

The aim of this systematic review is to determine whether HIV infection is associated with colonization or infection with antimicrobial-resistant bacteria.

\section{Methods}

The review is focused on bacterial pathogens causing infection or colonizing individuals with HIV infection. Mycobacteria and organisms causing sexually transmitted infections were excluded because this association has recently been reviewed [15] and because of the differences in transmission pathways for sexually transmitted infections.

Eligible publications were identified using the search strategy described in the Supplementary Material Table S2. The search strategy was applied to MEDLINE, EMBASE, Web of Science and LILACS databases up to week 3 of 2020. The African Journals Online and PubMed databases were searched for additional publications. The search was updated to June 2020. References of included studies and other reviews were manually searched for relevant publications. Articles identified were imported into the bibliographic software manager Endnote X7 (Clarivate Analytics, Philadelphia, PA, USA). Titles and abstracts were screened for eligibility by IDO and KK in duplicate.

Studies were included if they reported resistance data for colonization or infection by HIV status and stratified the results by bacterial species and antimicrobials tested. Colonization and infection were considered as defined by the authors of the respective studies. Studies describing species from the same family-such as Escherichia coli and Klebsiella pneumoniae (Enterobacteriaceae)-which did not present data by bacterial species were grouped in the analysis. No restriction with regards to geographical region, language or year of publication was applied. Case series were excluded. The review followed the PRISMA guidelines. The study protocol was registered with PROSPERO (CRD42020178907). The search strategy and analyses are described in the Supplementary Material.

Analyses were conducted using STATA (version 15, Stata-Corp, TX, USA). A descriptive analysis was performed for all eligible studies. If at least five studies reporting on the same organism (or group) and resistance to the same antimicrobial class were identified, then a random effects meta-analysis using the DerSimonian and Laird method was performed in STATA (using 'metan'). The meta-analyses were also stratified according carriage/colonization or infection. The pooled odds ratio estimates were grouped by organism, infection versus colonization and drug tested. Sensitivity analyses were performed to determine whether findings were consistent across different groups (Supplementary Material). Metaanalyses for proportions were performed using the package 'metaprop'. Heterogeneity of studies was evaluated using Cochrane recommendations [16] using the $I^{2}$ statistic. Heterogeneity was considered substantial if $I^{2}>50 \%$.

Completeness of reporting was assessed using the STROBE checklist [17], and for each item studies were graded into three categories: 'no information provided or unclear description', 'some information but insufficient', 'clear information provided'. The modified Newcastle-Ottawa scale was used to evaluate the quality of the studies [18].

\section{Results}

\section{Characteristics of identified studies}

Of 21489 unique citations identified by the search, 299 were selected for full-text review, of which 71 were suitable for inclusion in the main analysis. An additional 21 publications were identified from reference review. A total of 92 citations were included in the qualitative analysis (Fig. 1). Most studies were from either the United States ( $n=32,35 \%)$ or South Africa ( $n=21,23 \%)$. Fig. 2 shows where the studies were conducted. About half of the studies were conducted in low- and middle-income countries $(n=50,54 \%)$.

Most studies described resistance in a single pathogen $(n=83$, $90 \%$ ) while nine studies presented data for multiple organisms. AMR in Streptococcus pneumoniae was described in 28 studies (carriage $n=1$, infection $n=27$ ), Staphylococcus aureus in 47 (carriage $n=29$; infection $n=18$ ) and other organisms in 22 (carriage $n=6$; infection $n=16$ ). Studies in Enterobacteriaceae were on E. coli $(n=6)$, Salmonella spp. $(n=7)$, K. pneumoniae $(n=3)$, and Shigella spp. $(n=3)$. Supplementary Material Table S3 presents an overview of the studies included.

\section{Patient characteristics}

Studies included 40625 PLWH and 17187704 individuals without HIV. Most studies were conducted in inpatients $(n=44$, $48 \%$ ), or a combination of inpatients and outpatients ( $n=10,11 \%)$; 18 studies (20\%) reported on outpatients only, and six (7\%) on other settings such as prisons $(n=3)$, youth centres $(n=1)$, shelters $(n=1)$ and individuals recruited from the community $(n=1)$ (Supplementary Material Table S3). One study reported on isolates 


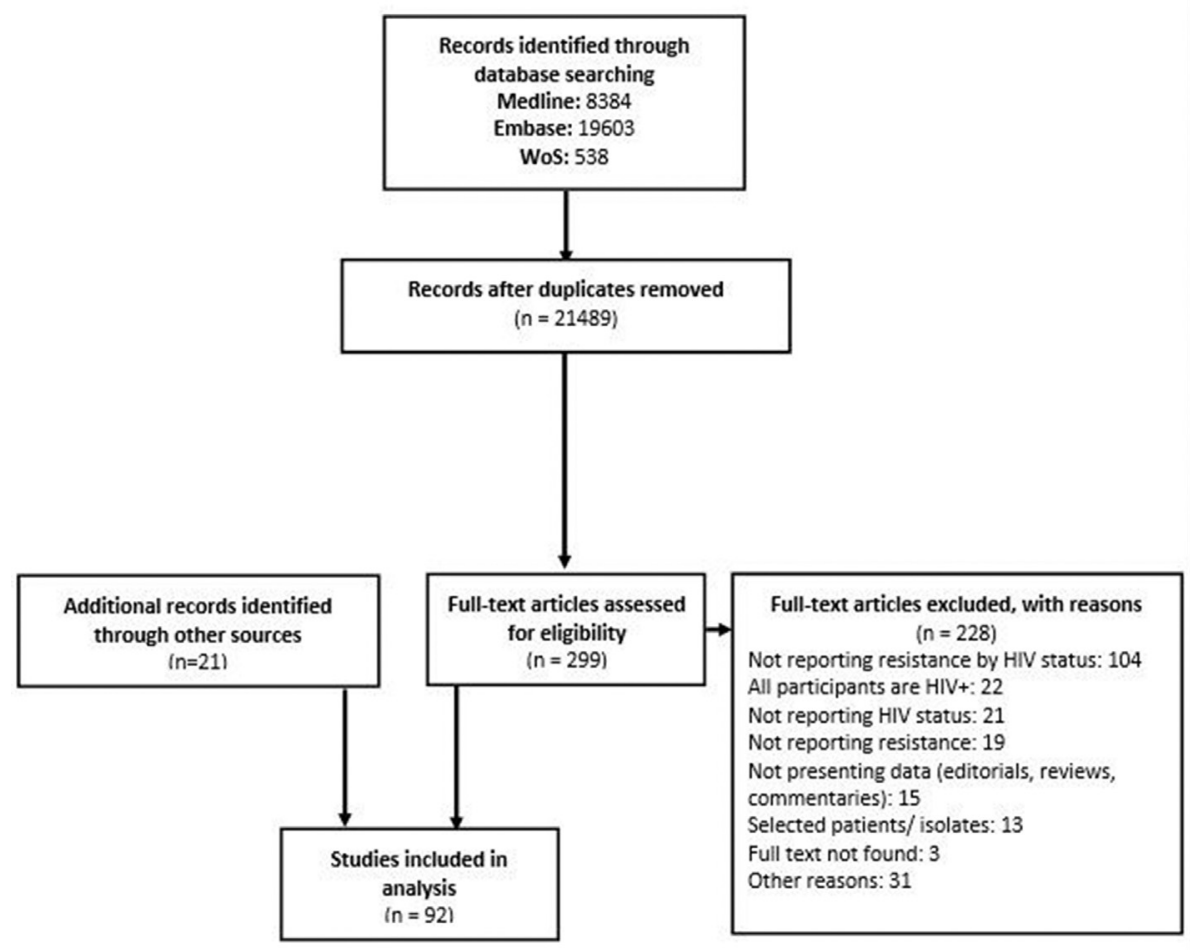

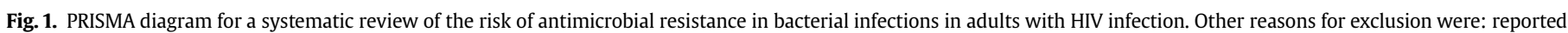

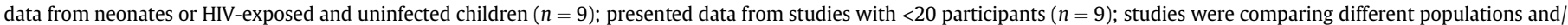

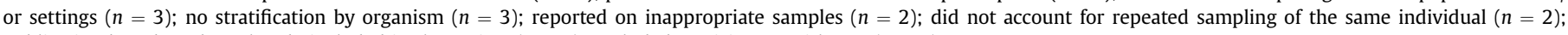
publication based on data already included in the review $(n=2)$; excluded participants with HIV $(n=1)$.

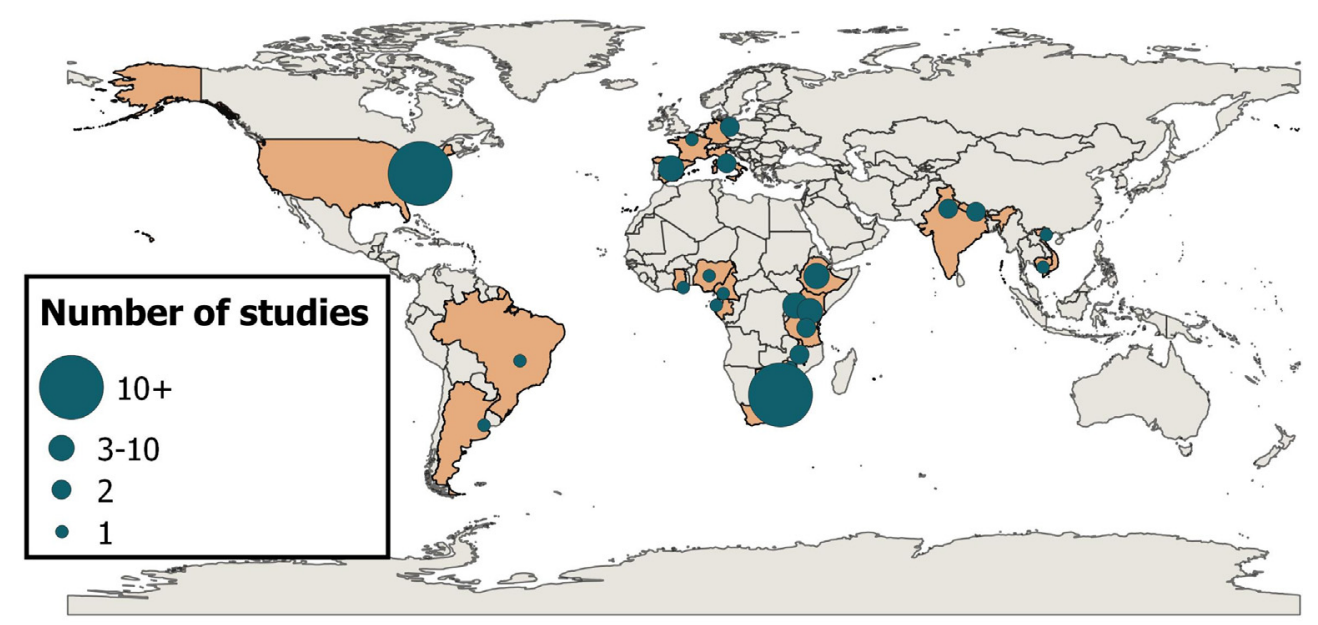

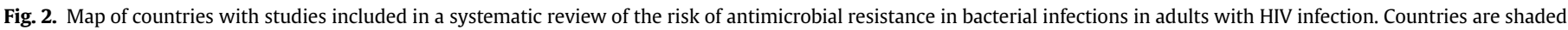

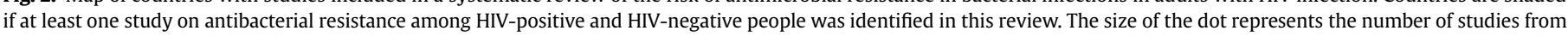
the respective country.

collected as part of laboratory surveillance and did not specify the source of the isolates; 13 studies did not provide details on the healthcare setting. Most of the studies were conducted in a single facility ( $n=54,59 \%)$.

Most studies reported data from adults ( $n=53,58 \%)$, while 15 (16\%) included only children and 22 (24\%) included both adults and children. For two studies, participant age was not reported.

HIV status was determined by performing an HIV test as part of the study procedures ( $n=26$ studies) or was obtained from patient records or as part of the medical history ( $n=52$ studies). In 14 studies, HIV status ascertainment was not clearly described.

Few studies reported on ART coverage ( $n=17,18 \%)$ and cotrimoxazole prophylaxis ( $n=22,24 \%$ ) among HIV-infected patients. CD4 cell counts at the time of the study were reported for 39 studies (42\%). Mean/median CD4 cell counts were $<200$ cells $/ \mu \mathrm{L}$ in 12 studies (31\%) and $\geq 200$ in 25 studies (64\%). For two studies the severity of the immunosuppression could not be determined. Forty-five (49\%) of the studies collected data prospectively, while 
43 (47\%) extracted data from medical records or laboratory databases; one study used both prospective and retrospective data collection, and three (3\%) did not specify how data collection was performed.

The pooled prevalence of resistance to selected antimicrobials by HIV status is shown in Table 1 and in Supplementary Material Figs S2 and S3.

\section{Antimicrobial resistance in Staphylococcus aureus}

Of the 47 studies describing AMR prevalence in S. aureus, 29 studies (62\%) focused on colonization only (nasal: $n=23$; multiple sites $n=6$ ), and $18(38 \%$ ) on infections (bacteraemia: $n=5$; skin and soft tissue infections: $n=5$; respiratory infections: $n=2$; multiple sites: $n=6$ ). Among studies evaluating methicillinresistant $S$. aureus (MRSA) colonization, six studies used methods enabling direct MRSA identification: chromogenic media $(n=5)$ and molecular methods $(n=1)$. Most studies reported predominantly on patients with community-acquired infections ( $n=10$, $56 \%$ ), while four (22\%) included patients with hospital-acquired infections and four (22\%) did not describe the type of infection. The studies included a total of 27364 PLWH and 17159190 individuals without HIV infection. Half of the studies (24/47) and 89\% of PLWH were from United States. Most studies (41/47, 87\%) were conducted after the introduction of widespread ART. The proportion of individuals on co-trimoxazole prophylaxis was not consistently reported ( $n=11,23 \%$ ) and ranged from 0 to $89 \%$.

PLWH had 2.12 (95\%CI 1.36-3.30) higher odds for colonization and 1.90 (95\%CI 1.45-2.48) higher odds for infection with MRSA compared with HIV-negative individuals. Eleven studies evaluated co-trimoxazole susceptibility of $S$. aureus isolates. The pooled OR for co-trimoxazole resistance was 1.40 (95\% CI $0.49-3.99)$ for colonization and 2.89 (95\%CI 0.48-17.37) for infection, comparing PLWH with HIV-negative individuals. Heterogeneity in ORs was high across studies ( $I^{2}$ range $82-98 \%$, Fig. 3c,d). Among individuals with $S$. aureus colonization, the odds of MRSA carriage were higher among PLWH than in those without HIV infection (Supplementary Material Fig. S4).

\section{Antimicrobial resistance in Streptococcus pneumoniae}

A total of 28 studies evaluated AMR in S. pneumoniae, including 9239 PLWH and 8368 individuals without HIV infection; South Africa contributed the majority of the HIV-positive patients (83\%). Studies were mostly conducted before the widespread availability of ART ( $n=18,64 \%)$. Reporting of ART $(1 / 28)$ and co-trimoxazole prophylaxis $(8 / 28)$ usage was very rare. Where described, the proportion of PLWH reported to take co-trimoxazole prophylaxis ranged between 0 and $67 \%$. More than two thirds of the studies described invasive pneumococcal disease $(n=20,71 \%)$, while isolates were cultured from multiple sample types in seven studies (30\%). One study reported on pneumococcal carriage.

Nine studies reported on co-trimoxazole resistance. S. pneumoniae isolates from PLWH had a 2.28 (95\%CI 1.75-2.97) higher odds of having decreased penicillin susceptibility compared to isolates from individuals without HIV. The pooled OR for cotrimoxazole resistance was 2.29 (95\%CI 1.31-4.00). Heterogeneity of studies was moderate to substantial $\left(I^{2} 60 \%\right.$ and $75 \%$, respectively, Fig. 3a,b).

\section{Antimicrobial resistance in Escherichia coli and Klebsiella pneumoniae}

Four studies described resistance in E. coli, two in K. pneumoniae, and one in both $E$. coli and $K$. pneumoniae isolates from patients presenting with infections. These studies included 849 HIVinfected and 12573 non-HIV-infected individuals. Isolates were from blood $(n=3)$, stool $(n=2)$, urine $(n=1)$ and respiratory samples $(n=1)$. The OR for the presence of resistance to thirdgeneration cephalosporins and co-trimoxazole in E. coli and K. pneumoniae causing infections was 1.59 (95\%CI 0.83-3.05) and 2.43 (95\%CI 1.36-4.32) respectively (Fig. 3e,f). Heterogeneity was high across studies for both third-generation cephalosporin resistance $\left(I^{2} 94 \%\right)$ and co-trimoxazole resistance $\left(I^{2} 78 \%\right)$.

\section{Quality of reporting in studies included}

Completeness of reporting according to the STROBE checklist was moderate in the majority of studies (66/92, 72\%) (Fig. 4). Sources of bias, sample size and generalizability of results were often not discussed. Also, most studies did not report on the number of participants or samples excluded from the analysis or the reasons for exclusion. When using the modified Newcastle-Ottawa Scale, most studies were of moderate $(n=30)$ or low $(n=57)$ quality. Only seven studies were of high quality.

\section{Discussion}

This review synthesizes for the first time data on AMR in PLWH compared to that in HIV-negative individuals. It shows that, for several important pathogens, PLWH are more likely to be infected or colonized with resistant bacterial strains than people without HIV infection. This holds true for two major Gram-positive and some Gram-negative organisms and for multiple antimicrobial classes. The higher prevalence of resistance among PLWH is of particular concern in low-income settings where alternative treatment options are limited by their availability and cost [19]. Cotrimoxazole may increase the risk of colonization with resistant Gram-negative and Gram-positive organisms [20-22]. Therefore, resistance is not surprising given the wide use of co-trimoxazole as

Table 1

Antimicrobial resistance to selected drugs

\begin{tabular}{|c|c|c|c|c|}
\hline \multirow[t]{2}{*}{ Organism } & \multirow[t]{2}{*}{ Antimicrobial } & \multirow{2}{*}{$\begin{array}{l}\text { Number of studies reporting } \\
\text { resistance by HIV status }\end{array}$} & \multicolumn{2}{|c|}{ Pooled prevalence of resistance $(95 \% \mathrm{CI})$} \\
\hline & & & $\mathrm{HIV}+$ & HIV- \\
\hline Staphylococcus aureus (infection) & MRSA & 14 & $49 \%(32-65)$ & $35 \%(25-44)$ \\
\hline \multirow[t]{2}{*}{ S. aureus (colonization) } & MRSA & 29 & $9 \%(7-11)$ & $6 \%(4-8)$ \\
\hline & Co-trimoxazole & 5 & $65 \%(44-86)$ & $55 \%(46-64)$ \\
\hline \multirow[t]{4}{*}{ Streptococcus pneumoniae (infection) } & Penicillin & 20 & $29 \%(23-36)$ & $15 \%(11-19)$ \\
\hline & Co-trimoxazole & 7 & $38 \%(30-47)$ & $20 \%(13-28)$ \\
\hline & Tetracyclines & 6 & $14 \%(8-20)$ & $19 \%(12-26)$ \\
\hline & Macrolides & 6 & $8 \%(4-12)$ & $8 \%(5-12)$ \\
\hline
\end{tabular}

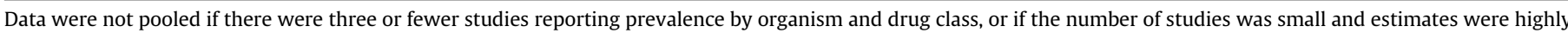

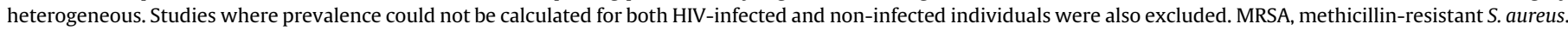



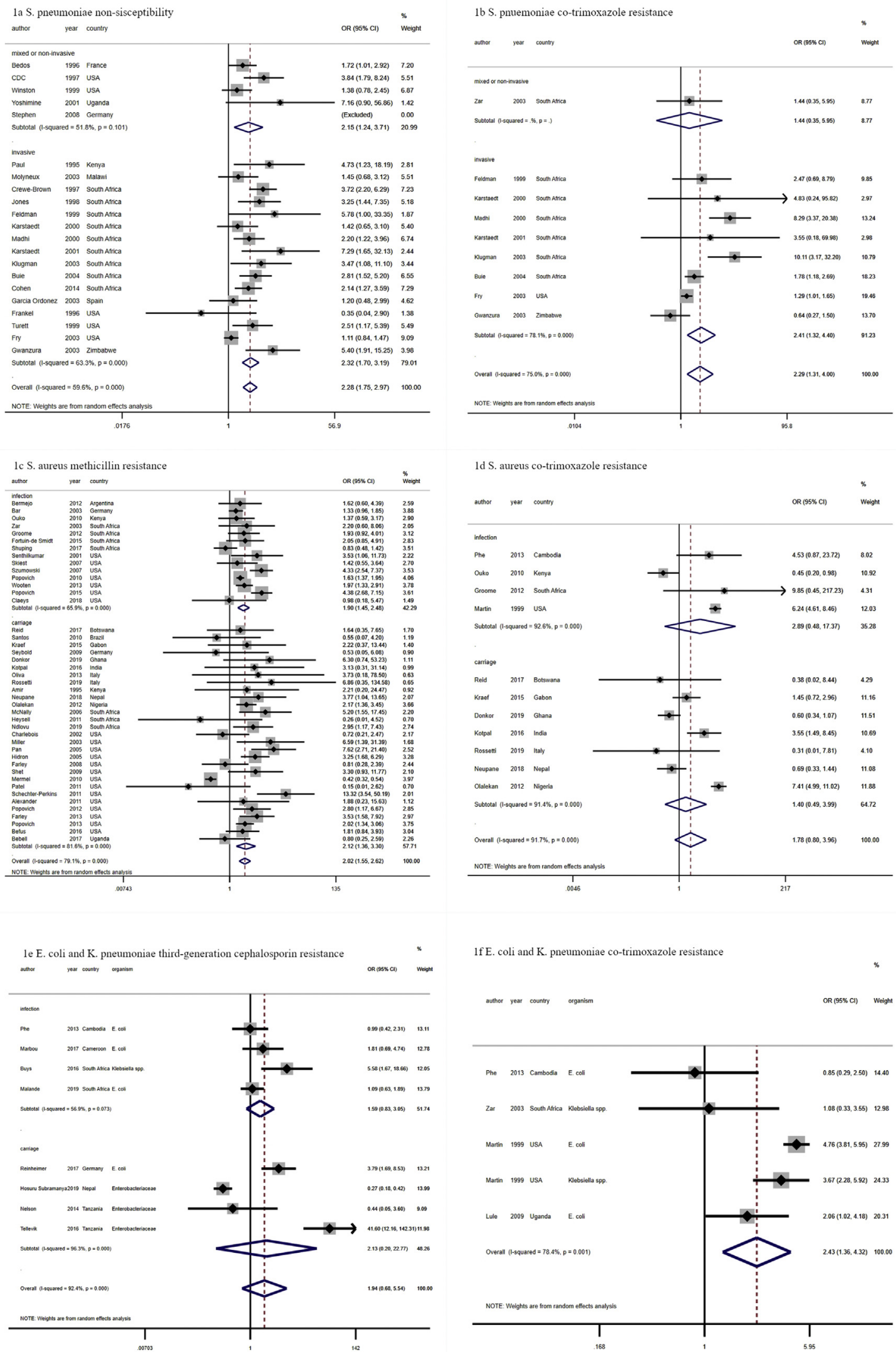

If E. coli and K. pneumoniae co-trimoxazole resistance

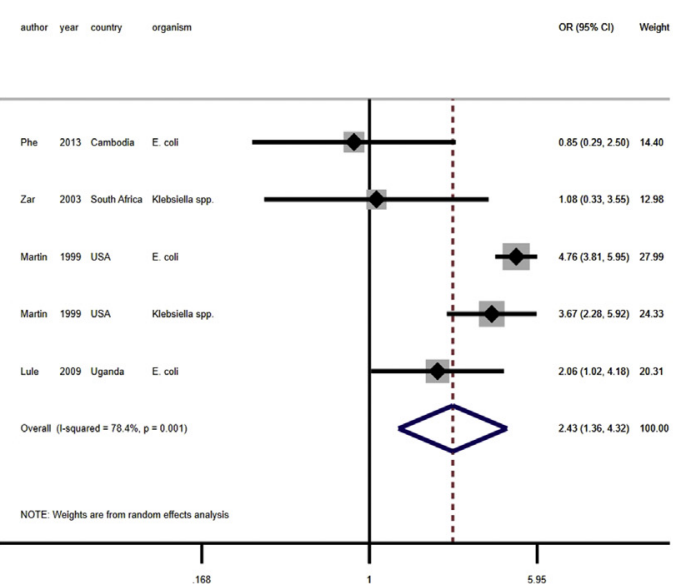

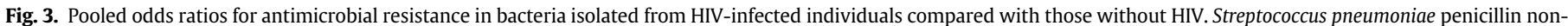

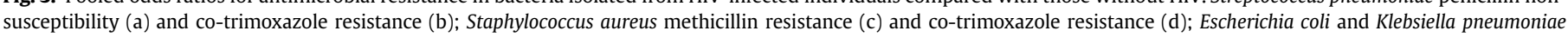
third-generation cephalosporin resistance (e) and co-trimoxazole resistance (f). 


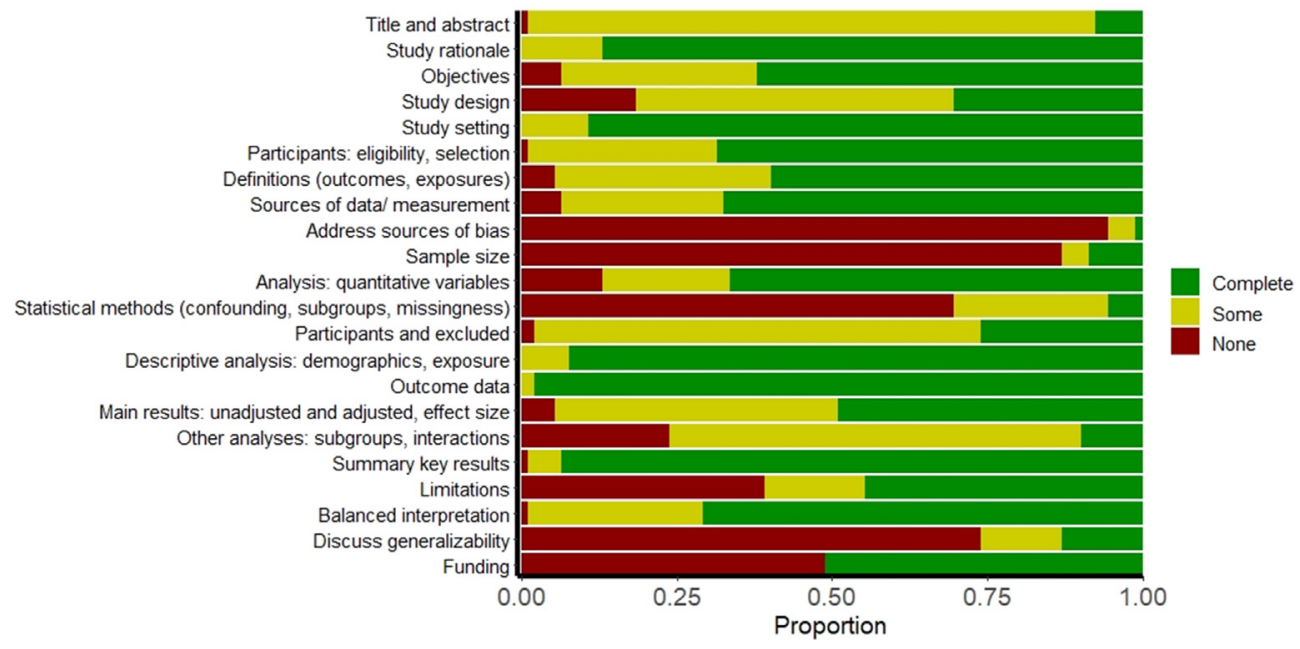

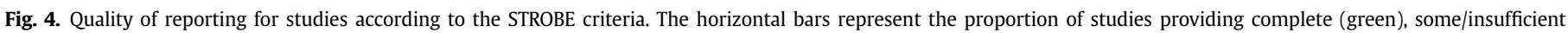
(yellow) or no/unclear (red) information on the respective items.

prophylaxis in PLWH in general; however, only a fraction of the studies presented data on co-trimoxazole use among their participants. The degree of immunosuppression and ART influence the risk of infection and hospitalization [23], but less than $20 \%$ of the studies reported the proportion of PLWH on ART, and only $41 \%$ described CD4 cell counts among PLWH.

Many penicillins and cephalosporins are readily available, inexpensive drugs used to treat infections caused by a wide variety of organisms. For $S$. aureus, the methicillin resistance gene (mecA) is especially problematic as it confers resistance not just to flucloxacillin but to almost all $\beta$-lactam drugs, including third-generation cephalosporins. Treatment of systemic MRSA infections is hence particularly challenging in low- and middle-income settings, as vancomycin and linezolid are not widely available and are expensive and difficult to use safely in PLWH.

This systematic review found that HIV infection is associated with methicillin resistance in $S$. aureus in studies predominantly from the United States and conducted prior to 2010. The steady decrease in MRSA infections in the United States since 2005 is attributed to the rigorous introduction of enhanced infection control programmes [24]. This decline was also observed in PLWH, although MRSA incidence remained higher than in the general population [25]. People who inject drugs and men who have sex with men are at increased risk for MRSA [26,27]. Given that these populations are more likely to be HIV-infected than the general population in the United States [28,29], the higher prevalence of MRSA may be due to confounding by these factors. However, the association between HIV infection and MRSA was also found in studies from sub-Saharan Africa [30] where heterosexual transmission of HIV is predominant, and therefore the association cannot be fully explained by this confounder. Previous studies have found that low CD4 cell counts and unsuppressed HIV viral load, injected drug use and identifying as a man who has sex with men are all associated with MRSA in PLWH [31,32]. The high prevalence of MRSA colonization in PLWH may be attributed to hospital admissions caused by HIV-related immunosuppression [33]. On the other hand, the association between HIV infection and resistance may be confounded by the presence of high-risk sexual activities [34]. Most studies in this review reported on community-acquired MRSA infections reflecting the widespread dissemination of successful MRSA clones.

This review found that the higher odds for MRSA colonization and infection among people with HIV was consistent across different levels of immunosuppression, ART use and country income, although some comparisons may be limited by the very small number of studies in the respective subgroups.

Co-trimoxazole resistance in $S$. aureus occurs either by mutations in the dihydrofolate reductase gene or by acquisition of variant dihydrofolate reductase genes by horizontal gene transfer [35]. While in Europe the prevalence of co-trimoxazole resistance in S. aureus is relatively low [36], this is not the case for sub-Saharan Africa and Asia [37,38]. The higher prevalence of resistance in these settings may be explained by the widespread use of co-trimoxazole and other sulphonamide-based drugs for treatment and prevention of infections [37,39], and potentially by the expansion of particular clonal types within the HIV population [30]. This is also reflected by the findings of this review which suggest an association between HIV and co-trimoxazole resistance, although estimates varied widely across studies.

S. pneumoniae is a major cause of pneumonia and meningitis; individuals with immunodeficiencies are at increased risk for invasive disease [40]. $\beta$-Lactam drugs, namely benzylpenicillin or amoxicillin, are the mainstay treatment for pneumococcal disease. Recent antimicrobial use, young age, previous hospitalization, infection with certain capsular serotypes, and geographical location are all risk factors for resistant $S$. pneumoniae strains causing invasive disease [41]. The epidemiology of S. pneumoniae nonsusceptible to penicillin varies geographically, and in the late 1990s the majority of invasive pneumococcal disease cases in the United States were attributed to pneumococcal conjugate vaccine (PCV7) serotypes [42].

Prevalence of co-trimoxazole resistance also varies geographically and is influenced by prior exposure either to co-trimoxazole [22] or to other sulphonamides such as sulfadoxine-pyrimethamine which are used in Africa for malaria treatment and preventive therapy in pregnancy [43].

Our review shows that HIV infection is associated with penicillin non-susceptibility and co-trimoxazole resistance in S. pneumoniae, with one in three isolates cultured from PLWH showing nonsusceptibility to penicillin and co-trimoxazole, although this may be overestimated by preferential sampling of people with HIV infection. Given that all studies presenting co-trimoxazole resistance data were conducted in sub-Saharan Africa, it is highly likely that a considerable proportion of PLWH had received cotrimoxazole prophylaxis. Most studies on S. pneumoniae were published before the ART roll-out, and therefore these results may not reflect the current situation. 
Our review identified only a small number of studies for the Enterobacteriaceae, but they suggest a higher odds of resistance to third-generation cephalosporins and co-trimoxazole in people with HIV infection, although the confidence interval crossed one for the former. Comparisons of AMR prevalence for other drug classes were precluded by the small number of studies identified. Given that third-generation cephalosporin resistance is associated with resistance to other drug classes, severe infections may require treatment with broad-spectrum antibiotics such as carbapenems. In contrast to high-income settings, where carbapenems usually are available but their use is restricted, these agents are usually unavailable in low-income settings or their use is cost-prohibitive.

Overall this systematic review shows an association between HIV infection and bacterial AMR across multiple pathogens and antimicrobial classes, although for co-trimoxazole, resistance may not be of clinical significance for some infections. Because of the diversity in study settings and populations, and the small number of studies identified, we were unable to assess the trend in AMR prevalence over time. Only 14 studies reported data collected after 2010 and five after 2015, thus precluding conclusions on the current epidemiology of AMR in these settings. Results were consistent across time periods, suggesting that the association between HIV infection and AMR is still relevant despite the relatively small number of more recent studies. This highlights the need for studies and surveillance to report on resistance prevalence according to HIV status, especially in studies from settings with a high HIV burden.

Despite the comprehensive search strategy, without restriction by language, year of publication and geography, only a small number of countries are represented in this review. For example, there were very few studies from South America and Asia. For individual pathogens, the majority of studies are from a single country; most of the studies of $S$. aureus were from the USA, and most of the studies of $S$. pneumoniae were from South Africa. Consequently, the results of this review may not be generalizable beyond these countries. Despite the high burden of HIV in subSaharan Africa, there are few data on HIV and AMR for most countries apart from South Africa. Additionally, there was substantial heterogeneity among studies which is not surprising given the differences in settings, patient populations, sample types and laboratory methods used for testing. Further heterogeneity may have been introduced by preferential sampling of people with HIV leading to more frequent diagnosis of infection as compared to individuals uninfected with HIV. The high heterogeneity between studies may impact on the generalizability of these findings. For most pathogens, a meta-analysis could not be performed because of the small number of studies. Because this review included observational studies, findings may have been affected by confounding.

This is, to our knowledge, the first systematic review evaluating the association between HIV infection and AMR in a wide range of bacterial pathogens. A comprehensive assessment of the quality of reporting was performed using the STROBE checklist.

The findings of this review underscore the need for further studies evaluating the association between HIV and AMR, especially from countries with a high HIV burden [7]. Most studies included were published before 2010 and were of moderate and low quality, which may affect the validity of our findings. This study further highlights the need for a better understanding of AMR prevalence in low-income settings and on the extent to which people with HIV may be at increased risk for infections with resistant bacterial pathogens, emphasizing the need for improved antimicrobial resistance testing and reporting. Increasing diagnostic capacity and AMR surveillance, as well as promoting further research into AMR and HIV, could ultimately lead to improved management of HIV-infected patients.

\section{Transparency declaration}

The authors declare that they have no conflicts of interest. IDO received funding though the Wellcome Trust Clinical PhD Programme awarded to the London School of Hygiene \& Tropical Medicine (grant number 203905/Z/16/Z).

\section{Appendix A. Supplementary data}

Supplementary data to this article can be found online at https://doi.org/10.1016/j.cmi.2021.03.026.

\section{References}

[1] Cosgrove SE. The relationship between antimicrobial resistance and patient outcomes: mortality, length of hospital stay, and health care costs. Clin Infect Dis 2006;42(Suppl 2):S82-9.

[2] Holmes AH, Moore LS, Sundsfjord A, Steinbakk M, Regmi S, Karkey A, et al. Understanding the mechanisms and drivers of antimicrobial resistance. Lancet 2016:387:176-87.

[3] Chatterjee A, Modarai M, Naylor NR, Boyd SE, Atun R, Barlow J, et al. Quantifying drivers of antibiotic resistance in humans: a systematic review. Lancet Infect Dis 2018;18:e368-78.

[4] UNAIDS., UNAIDS. Data. Available from: https://www.unaids.org/sites/default/ files/media_asset/2019-UNAIDS-data_en.pdf; 2019.

[5] Sogaard OS, Lohse N, Gerstoft J, Kronborg G, Ostergaard L, Pedersen C, et al. Hospitalization for pneumonia among individuals with and without HIV infection, 1995-2007: a Danish population-based, nationwide cohort study. Clin Infect Dis 2008;47:1345-53.

[6] Morgan E, Hohmann S, Ridgway JP, Daum RS, David MZ. Decreasing incidence of skin and soft-tissue infections in 86 US Emergency Departments, 2009-2014. Clin Infect Dis 2019;68:453-9.

[7] Ford N, Shubber Z, Meintjes G, Grinsztejn B, Eholie S, Mills EJ, et al. Causes of hospital admission among people living with HIV worldwide: a systematic review and meta-analysis. Lancet HIV 2015;2:e438-44.

[8] Maciel RA, Kluck HM, Durand M, Sprinz E. Comorbidity is more common and occurs earlier in persons living with HIV than in HIV-uninfected matched controls, aged 50 years and older: a cross-sectional study. Int J Infect Dis 2018; $70: 30-5$

[9] Guaraldi G, Orlando G, Zona S, Menozzi M, Carli F, Garlassi E, et al. Premature age-related comorbidities among HIV-infected persons compared with the general population. Clin Infect Dis 2011;53:1120-6.

[10] Kong AM, Pozen A, Anastos K, Kelvin EA, Nash D. Non-HIV comorbid conditions and polypharmacy among people living with HIV age 65 or older compared with HIV-negative individuals age 65 or older in the United States: a retrospective claims-based analysis. AIDS Patient Care STDS 2019;33:93-103.

[11] Martin-Iguacel R, Pedersen C, Llibre JM, Sondergaard J, Ilkjaer FV, Jensen J, et al. Prescription of antimicrobials in primary health care as a marker to identify people living with undiagnosed HIV infection, Denmark, 1998 to 2016. Euro Surveill 2019;24.

[12] World Health Organization. Consolidated guidelines on the use of antiretroviral drugs for treating and preventing HIV infection. Recommendations for a public health approach. Geneva, Switzerland: World Health Organization; 2016. Available from: https://www.who.int/publications/guidelines/hiv_aids/en/.

[13] European AIDS Clinical Society. Guidelines. Version 10.0. Available from: https://www.eacsociety.org/files/guidelines-10.0_final_2_2.pdf; 2019.

[14] Suthar AB, Vitoria MA, Nagata JM, Anglaret X, Mbori-Ngacha D, Sued O, et al. Co-trimoxazole prophylaxis in adults, including pregnant women, with HIV: a systematic review and meta-analysis, Lancet HIV 2015;2:e137-50.

[15] Pradipta IS, Forsman LD, Bruchfeld J, Hak E, Alffenaar JW. Risk factors of multidrug-resistant tuberculosis: a global systematic review and meta-analysis. J Infect 2018;77:469-78.

[16] The Cochrane Collaboration. Cochrane handbook for systematic reviews of interventions, Version 5.1.10. Available from: https://handbook-5-1.cochrane. org/front_page.htm; 2011.

[17] von Elm E, Altman DG, Egger M, Pocock SJ, Gotzsche PC, Vandenbroucke JP, et al. The Strengthening the Reporting of Observational Studies in Epidemiology (STROBE) statement: guidelines for reporting observational studies. Lancet 2007:370:1453-7.

[18] Wells GA, Shea B, O'Connell D, Peterson J, Welch V, Losos M, et al. The Newcastle-Ottawa Scale (NOS) for assessing the quality of nonrandomised studies in meta-analyses. Available from: http://www.ohri.ca/programs/ clinicalepidemiology/oxford.asp.

[19] Frost I, Craig J, Joshi J, Faure K, Laxminarayan R. Access barriers to antibiotics. Washington, DC: Center for Disease Dynamics, Economics \& Policy; 2019. Available from: https://cddep.org/wp-content/uploads/2019/04/AccessBarrier stoAntibiotics_CDDEP_FINAL.pdf.

[20] Chiller TM, Polyak CS, Brooks JT, Williamson J, Ochieng B, Shi YP, et al. Daily trimethoprim-sulfamethoxazole prophylaxis rapidly induces corresponding 
resistance among intestinal Escherichia coli of HIV-infected adults in Kenya. I Int Assoc Phys AIDS Care (Chic) 2009;8:165-9.

[21] D'Souza AW, Moodley-Govender E, Berla B, Kelkar T, Wang B, Sun X, et al. Cotrimoxazole prophylaxis increases resistance gene prevalence and $\alpha$-diversity but decreases $\beta$-diversity in the gut microbiome of HIV-exposed, uninfected infants. Clin Infect Dis 2020;71:2858-68.

[22] Mwenya DM, Charalambous BM, Phillips PP, Mwansa JC, Batt SL, Nunn AJ, et al. Impact of cotrimoxazole on carriage and antibiotic resistance of Streptococcus pneumoniae and Haemophilus influenzae in HIV-infected children in Zambia. Antimicrob Agents Chemother 2010;54:3756-62.

[23] Bellino S, Borghetti A, Lombardi F, Camoni L, Ciccullo A, Baldin G, et al. Trends of hospitalisations rates in a cohort of HIV-infected persons followed in an Italian hospital from 1998 to 2016. Epidemiol Infect 2019;147:e89.

[24] Kourtis AP, Hatfield K, Baggs J, Mu Y, See I, Epson E, et al. Vital signs: epidemiology and recent trends in methicillin-resistant and in methicillinsusceptible Staphylococcus aureus bloodstream infections - United States. MMWR Morb Mortal Wkly Rep 2019;68:214-9.

[25] Delorenze GN, Horberg MA, Silverberg MJ, Tsai A, Quesenberry CP, Baxter R. Trends in annual incidence of methicillin-resistant Staphylococcus aureus (MRSA) infection in HIV-infected and HIV-uninfected patients. Epidemiol Infect 2013;141:2392-402.

[26] Young DM, Harris HW, Charlebois ED, Chambers H, Campbell A, PerdreauRemington F, et al. An epidemic of methicillin-resistant Staphylococcus aureus soft tissue infections among medically underserved patients. Arch Surg 2004;139:947-51. discussion 951-3.

[27] David MZ, Daum RS. Community-associated methicillin-resistant Staphylococcus aureus: epidemiology and clinical consequences of an emerging epidemic. Clin Microbiol Rev 2010;23:616-87.

[28] Centers for Disease Control and Prevention (CDC). HIV prevalence, unrecognized infection, and HIV testing among men who have sex with men-five U.S. cities, June 2004-April 2005. MMWR Morb Mortal Wkly Rep 2005;54:597-601.

[29] Nelson KE, Galai N, Safaeian M, Strathdee SA, Celentano DD, Vlahov D. Temporal trends in the incidence of human immunodeficiency virus infection and risk behavior among injection drug users in Baltimore, Maryland, 1988-1998. Am J Epidemiol 2002;156:641-53.

[30] Olalekan AO, Schaumburg F, Nurjadi D, Dike AE, Ojurongbe O, Kolawole DO, et al. Clonal expansion accounts for an excess of antimicrobial resistance in Staphylococcus aureus colonising HIV-positive individuals in Lagos, Nigeria. Int J Antimicrob Agents 2012;40:268-72.

[31] Mathews WC, Caperna JC, Barber RE, Torriani FJ, Miller LG, May S, et al. Incidence of and risk factors for clinically significant methicillin-resistant Staphylococcus aureus infection in a cohort of HIV-infected adults. J Acquir Immune Defic Syndr 2005;40:155-60.
[32] Hidron AI, Moanna A, Rimland D. The rise and fall of methicillin-resistant Staphylococcus aureus infections in HIV patients. AIDS 2011;25:1001-3.

[33] Kluytmans J, van Belkum A, Verbrugh H. Nasal carriage of Staphylococcus aureus: epidemiology, underlying mechanisms, and associated risks. Clin Microbiol Rev 1997;10:505-20.

[34] Crum-Cianflone NF, Shadyab AH, Weintrob A, Hospenthal DR, Lalani T, Collins G, et al. Association of methicillin-resistant Staphylococcus aureus (MRSA) colonization with high-risk sexual behaviors in persons infected with human immunodeficiency virus (HIV). Medicine (Baltimore) 2011;90: $379-89$.

[35] Foster TJ. Antibiotic resistance in Staphylococcus aureus. Current status and future prospects. FEMS Microbiol Rev 2017;41:430-49.

[36] den Heijer CD, van Bijnen EM, Paget WJ, Pringle M, Goossens $H$, Bruggeman CA, et al. Prevalence and resistance of commensal Staphylococcus aureus, including meticillin-resistant S. aureus, in nine European countries: a cross-sectional study. Lancet Infect Dis 2013:13:409-15.

[37] Nurjadi D, Olalekan AO, Layer F, Shittu AO, Alabi A, Ghebremedhin B, et al. Emergence of trimethoprim resistance gene dfrG in Staphylococcus aureus causing human infection and colonization in sub-Saharan Africa and its import to Europe. J Antimicrob Chemother 2014;69:2361-8.

[38] Nurjadi D, Schafer J, Friedrich-Janicke B, Mueller A, Neumayr A, Calvo-Cano A, et al. Predominance of $\mathrm{dfrG}$ as determinant of trimethoprim resistance in imported Staphylococcus aureus. Clin Microbiol Infect 2015;21:1095 e5-9.

[39] Cornick JE, Harris SR, Parry CM, Moore MJ, Jassi C, Kamng'ona A, et al. Genomic identification of a novel co-trimoxazole resistance genotype and its prevalence amongst Streptococcus pneumoniae in Malawi. J Antimicrob Chemother 2014;69:368-74.

[40] van Aalst M, Lotsch F, Spijker R, van der Meer JTM, Langendam MW Goorhuis A, et al. Incidence of invasive pneumococcal disease in immunocompromised patients: a systematic review and meta-analysis. Travel Med Infect Dis 2018;24:89-100.

[41] Kim L, McGee L, Tomczyk S, Beall B. Biological and epidemiological features of antibiotic-resistant Streptococcus pneumoniae in pre- and post-conjugate vaccine eras: a United States perspective. Clin Microbiol Rev 2016;29: $525-52$.

[42] Kyaw MH, Lynfield R, Schaffner W, Craig AS, Hadler J, Reingold A, et al. Active bacterial core surveillance of the emerging infections program, effect of introduction of the pneumococcal conjugate vaccine on drug-resistant Streptococcus pneumoniae. N Engl J Med 2006;354:1455-63.

[43] Feikin DR, Dowell SF, Nwanyanwu OC, Klugman KP, Kazembe PN, Barat LM, et al. Increased carriage of trimethoprim/sulfamethoxazole-resistant Streptococcus pneumoniae in Malawian children after treatment for malaria with sulfadoxine/pyrimethamine. J Infect Dis 2000;181:1501-5. 